\title{
Physiological responses to the special judo fitness test in elite Spanish judo athletes: a new monitoring approach
}

\author{
Cristina CASALS ${ }^{* 1,2}$, Jesús R. HUERTAS ${ }^{1,2}$, Yaira BARRANCO-RUIZ ${ }^{3}$, \& Raquel ESCOBAR-MOLINA ${ }^{2}$ \\ ${ }_{1}^{1}$ Institute of Nutrition and Food Technology - University of Granada (Spain) \\ ${ }^{2}$ Faculty of Sport Sciences -University of Granada (Spain) \\ ${ }^{3}$ School of Physical Culture - National University of Chimborazo (Ecuador)
}

\section{5th IMACSSS World Scientific Congress Abstracts, Rio Maior (Portugal), October 6-8 Section: Biological, kinesiological and physiological aspects of MA\&CS Type: Oral communication}

\section{Introduction}

The physiological assessment of the elite athlete is of critical importance for his/her health and competitive success. In judo, specific tests are required because the anaerobic capacity is responsible for the explosive scoring actions in combat, while the aerobic capacity is also essential for recovery during and between matches (Franchini, et al, 2013). In this regard, the Special Judo Fitness Test (SJFT) was developed by Sterkowicz (1995), and it is one of the most used tests in judo (Drid, et al, 2012), probably explained by its easy applicability and its specificity that makes the test more appropriate for the judoka evaluation than a Wingate test (Szmuchrowski, et al, 2013). However, the test only considers a few performance indicators; thus, the aim of our study was to assess additional physiologic markers during the SJFT execution in elite judo athletes.

\section{Methodology}

Forty athletes from the Spanish National Judo Teams participated in this study, 10 males (MS) and 10 females (FS) competed in senior division, while 10 males (MJ) and 10 females (MJ) competed in junior division. During a competitive period, all athletes performed the SJFT, in which three athletes of similar body mass are needed: one participant (tori) is evaluated, and the other two (ukes) receive throws. The tori begins the test between the two ukes ( $3 \mathrm{~m}$ away from each uke). On a signal, the tori runs to one of the ukes and applies a throwing technique called ippon-seoi-nage (one shoulder throw) completing as many throws as possible within the test time. The SJFT is composed of three parts $(15,30$, and 30 seconds) separated by 10 -seconds recovery periods. The SJFT index was calculated as: Index $=$ (Hear rate after + Heart rate 1 min after the test $) /$ Total number of throws. Additionally, some micro-invasive (lactate and hematocrit) and non-invasive (fatigue index) markers were included and normalized in order to obtain values ranging from 0 to 100 (with 100 as the maximum performance level). Blood sampling was performed at rest, 1 min and $15 \mathrm{~min}$ after the test by capillary puncture. Fatigue index was calculated according to the number of throws executed in the third part of the SJFT compared to those of previous phases. The SJFT index, total number of throws, and heart rate recoveries were also normalized, then we obtained several performance indicators that can be displayed in a 6-axischart with visually attractive and utility for the coach. Data is presented as mean \pm standard error. Statistical comparisons by age category and sex were performed using a 2-way analysis of variance followed, when significant $(\mathrm{p}<0.05)$, by Bonferroni post hoc test. The proposed index (the mean of the 6 normalized variables) was correlated with the classical SJFT index by using the Pearson's correlation coefficient. All analyses were conducted using the SPSS statistical package. This study obtained ethical approval from the Research Ethics Committee of the University of Granada and was in accordance with the Helsinki declaration. 


\section{Results}

The classical SJFT index was $13.34 \pm 0.38$ in MS, $12.58 \pm 0.38$ in MJ, $12.55 \pm 0.38$ in FS, and $13.21 \pm 0.38$ in FJ. Our proposed index was $63.1 \pm 5.1 \%$ in MS, $69.9 \pm 5.1 \%$ MJ, $61.5 \pm 5.1 \%$ in FS, and $65.6 \pm 5.1 \%$ in FJ, and was significantly correlated with the classical SJFT index $(r=0.75, p<0.001)$. There were no statistically significant differences by sex and age. Among the analyzed variables, SM should improve their anaerobic capacity since their normalized number of throws was $40 \%$ while presented good fatigue resistance (85\%). Therefore, SM executed a low number of throws $(25.9 \pm 0.54)$ but they maintained the same load in the third SJFT part. Moreover, FS and FJ should improve their aerobic capacity, the normalized heart rate recovery was $43 \%$ and $26 \%$ respectively, showing that most of the female athletes recovered less than $30 \mathrm{bpm}$ in the first minute after the short duration, high-intensity intermittent effort.

\section{Discussion and conclusion}

Our results showed different needs between the judoka groups where, during a competitive period, MJ showed best SJFT performance than the other groups with no statistically significant differences. As mentioned before, the SJFT is of great utility because its simplicity and great reliability. Nevertheless, at the elite level, it is often convenient to obtain a more complete assessment of the judoka. Our new approach to the SJFT can be a useful tool for coaches, especially when accompanied by the 6-axis chart with individual graphical representations. The normalized variables from 0 to 100 could make easier the result interpretation for coaches and athletes. Although this approach might not be applied so frequently and widely as the classical SJFT, due to its invasive characteristic, both lactates and hematocrits can be determined through capillary blood by finger stick or from the earlobe, and analyzed with portable equipment. Our results highlighted that elite Spanish judokas should improve the anaerobic capacity in the case of SM, and the aerobic capacity in FS and, especially, in FJ. These training adjustments can be programmed using the SJFT results and completed with this new approach.

\section{References}

Drid, P., Trivic, T., \& Tabakov, S. (2012). Special judo fitness test - a review. Serbian Journal of Sports Sciences, 6(4), 119-27.

Franchini, E., Artioli, G.G., \& Brito, C.J. (2013). Judo combat: time-motion analysis and physiology. International Journal of Performance Analysis in Sport, 13(3), 624-641.

Szmuchrowski, L.A., Rodrigues, S.A., Corgosinho, R.F., Pinheiro, G.S., Pedrosa, G.F., Motta Drummond, M.D., et al (2013). Correlation between the performance in the Special Judo Fitness Test abd the Wingate Anaerobic Test. Archives of Budo, 9(3), 175-179.

Sterkowicz, S. (1995). Test specjalnejsprawnoscituchowej w judo. Antropomotoyka, 12-13, 29-44.

Key words: Sports performance; elite athletes; martial arts; combat sports; judo; SJFT.

Study supported by the Consejo Superior de Deportes - Ministerio de Educación y Ciencia [Higher Council of Sports, Spanish Ministry of Education and Science] (33/UPB10/10). 